\title{
Propuesta metodológica para el desarrollo de productos desechables catalogados como sustentables en México
}

\author{
Ing. Carlos E Vázquez Peña ${ }^{a}$, Dr. David Cortés Sáenz ${ }^{b}$ \\ ${ }^{a}$ Ing. en Aeronáutica por la UACJ, estudiante de la maestría en diseño de producto en la UACJ, \\ carlos.e.vazquez.75@gmail.com,${ }_{2}$ b Dr. en proyectos de innovación tecnológica, coordinador de la \\ maestría en diseño de producto en laUACJ, david.cortes@uacj.mx
}

\section{Resumen}

Actualmente los efectos del impacto ambiental son evidentes. Las decisiones de inversión tanto de los productores como de los países, y el comportamiento que se tiene por parte de los consumidores, siguen siendo ampliamente focalizados a resolver problemas locales y a corto plazo. El diseño ha fomentado este tipo de modelo de consumo, dando un énfasis a crear productos para un mercado donde las alternativas sustentables han recibido poca atención. Un ejemplo claro son los desechables, utilizados para el transporte de alimentos.

Esta investigación tiene como objetivo identificar aspectos de metodologías existentes, para desarrollar una propuesta aplicable a los productos desechables utilizados en el sector alimenticio en México y con un enfoque al ciclo de vida del producto y la economía circular.

Palabras clave: Ecodiseño, ACV, economía circular, diseño de producto

\begin{abstract}
The effect of environmental impact around the glove is now evident. Inversion decisions from countries and companies and the behavior of consumers are still widely focalized and directed to solve problems localized and in the short term. In this context, design and designers had been part of this consumption model, empathizing on the creation of products in a market, where the sustainable alternatives had a low presence. One example is the disposable products in the food industry.

This research aims to identify aspects of existing methodologies, to develop a proposal applicable to disposable products used in the food sector in Mexico and with a focus on the product life cycle and the circular economy.
\end{abstract}

Keywords: Ecodesign, Life Cycle Assessment, circular economy, product design 


\section{Introducción}

Durante el siglo XX el diseño de productos ha servido para desarrollar el mercado global, buscando dar soluciones a las problemáticas y así mejorar las condiciones de vida de las personas. Para esto, las cadenas de producción global han sido fundamentales, logrando disminuir el costo de los productos y ampliando las redes de distribución. Sin embargo, la búsqueda del crecimiento económico ha restado importancia al impacto de las actividades humanas en el ecosistema (Bastidas Pacheco \& Hernández, 2019).

A partir de los años 70's y con un creciente auge en lo que va del siglo XXI, se ha fortalecido la toma de conciencia y la realización de acciones que pretenden disminuir el impacto al ecosistema (García \& Cárcova, 2019). La Organización de las Naciones Unidas (ONU) a través del Programa de las Naciones Unidas para el Desarrollo (PNUD) ha establecido 17 objetivos para el desarrollo sostenible, los cuales se muestran en la Fig. 1 (United Nations Development Programme, 2015). Estos objetivos son la base del "plan para una ciudad sostenible Juárez 2030" (Gobierno Municipal de Ciudad Juárez et al., 2018). En donde se denotan acciones específicas a nivel local.

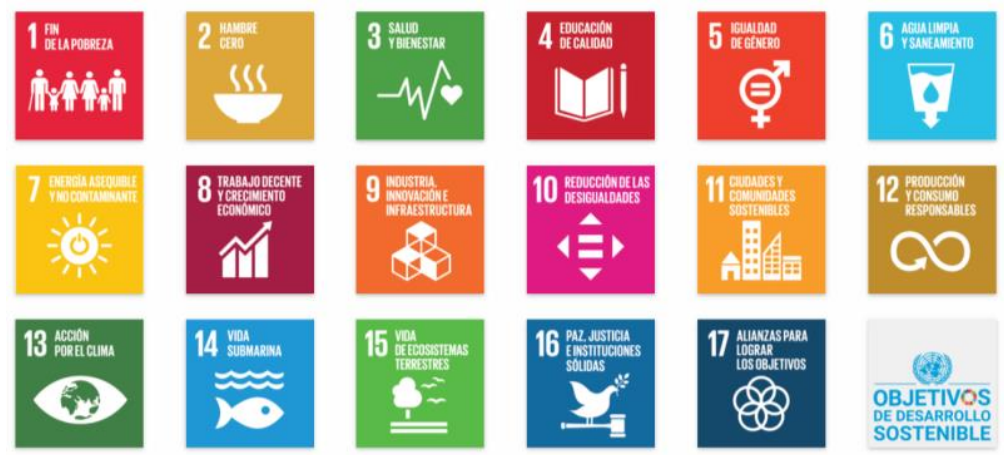

Fig. 1 Objetivos para el desarrollo sostenible (United Nations Development Programme, 2015)

Esta investigación parte del objetivo número 12, en el cual se propone buscar medios productivos y de consumo responsables con el medio ambiente, aportando a las siguientes metas:

- Para el 2030, reducir la generación de los desechos a través de actividades de prevención, reducción, reciclado y reutilización

- Ayudar a los países a fortalecer su capacidad científica y tecnológica para crear modalidades de consumo y producción que sean más sostenibles.

Dentro del "plan para una ciudad sostenible Juárez 2030", se crearon indicadores que buscan cumplir las metas descritas por la ONU a través de actividades de prevención, 
reducción, reciclado y reutilización. De estos indicadores son relevantes para esta investigación los siguientes (Gobierno Municipal de Ciudad Juárez et al., 2018):

- $\quad$ 12.1.1 Reducir a $1 \mathrm{~kg}$ o menos la cantidad de basura recolectada per cápita.

- 12.1.2 Incrementar al 100\% el porcentaje de basura del relleno sanitario que se recicla y que se reutiliza.

- $\quad$ 12.1.3 Incrementar al $80 \%$ el porcentaje de viviendas particulares habitadas que separa sus residuos en orgánicos e inorgánicos para su entrega al servicio público de recolección o en un contenedor.

El objetivo del presente artículo, es identificar aspectos claves en distintas metodologías existentes, los cuales puedan ser utilizados en una propuesta que este dirigida a los productos desechables.

\section{Contaminación}

A través de las actividades que realiza el hombre se pueden encontrar distintos tipos de desechos, dentro de los cuales se encuentran los residuos sólidos urbanos. En 2015 se generaron aproximadamente 31.5 millones de toneladas en México, mostrando un incremento del 25\% desde el 2003. De estas, solo el 5\% fue reciclado (INECC, 2018; SEMARNAT, 2016), y el resto termina en los rellenos sanitarios municipales.

La secretaria de desarrollo urbano del estado de chihuahua realizó un diagnóstico para conocer la situación del manejo de residuos sólidos urbanos en los distintos municipios que conforman esta entidad. En dicho estudio, se encontró que en Ciudad Juárez se generaron 1,200 toneladas diarias y solo 199 toneladas de basura pasaban a un centro de reciclado (INECC, 2018). Dentro de estos desperdicios se pueden encontrar una cantidad significativa de artículos desechables, los cuales después de un corto tiempo de uso (al finalizar la comida o terminar una bebida) son desechados en la basura.

\section{Economía circular y análisis de ciclo de vida.}

Hablar de economía circular refiere al uso de sistemas donde se busca que la materia prima, productos, procesos y desechos, puedan ser Repensados, Rediseñados, Refabricados, Reparados, Redistribuidos, Reducidos, Reutilizados, Reciclados y Recuperados (conocidas como las "9 $R$ ' $s$ "). Algunos beneficios que se obtienen al utilizar la economía circular son: la reducción del uso de materiales y energía nuevos, la reducción de desperdicios y emisiones, nuevas oportunidades de empleo, la reutilización de los recursos, entre otros. (Korhonen, Honkasalo, \& Seppälä, 2018).

El análisis de ciclo de vida (ACV) es un método que sirve para cuantificar la huella medioambiental de un producto, en el cual se toma en consideración el material, flujo de energía durante cada paso de su ciclo de vida. Este tipo de análisis produce un estimado del 
consumo de energía, emisiones de gases de efecto invernadero (GEI), y métricos de otros impactos medioambientales (Snyder, 2016). Algunas ventajas de la aplicación de este método, según lo expuesto por María Bernatene (2019), son:

- La identificación de procesos donde ocurren el mayor uso de materiales, energía y emisiones, buscando implementar mejoras.

- El análisis del proceso del producto desde "la cuna" hasta su desecho.

- El Lograr diferenciar entre una diseño sustentable y estrategias superficiales.

- Permitir ahorros, tanto de material, recursos económicos y abaratamiento de los costos.

- Es necesario si se desea implementar eco etiquetado y declaraciones ambientales.

\section{2. metodologías existentes}

Para formular una metodología, es necesario partir de propuestas ya existentes, como la de Ulrich y Eppinger (2003) mostrada en la Fig. 2, en donde se describe un proceso lineal y se presentan las etapas clásicas del desarrollo de un producto. Esta es un punto de referencia, siendo crucial incluir aspectos ambientales donde se considere el ciclo de vida en su totalidad.

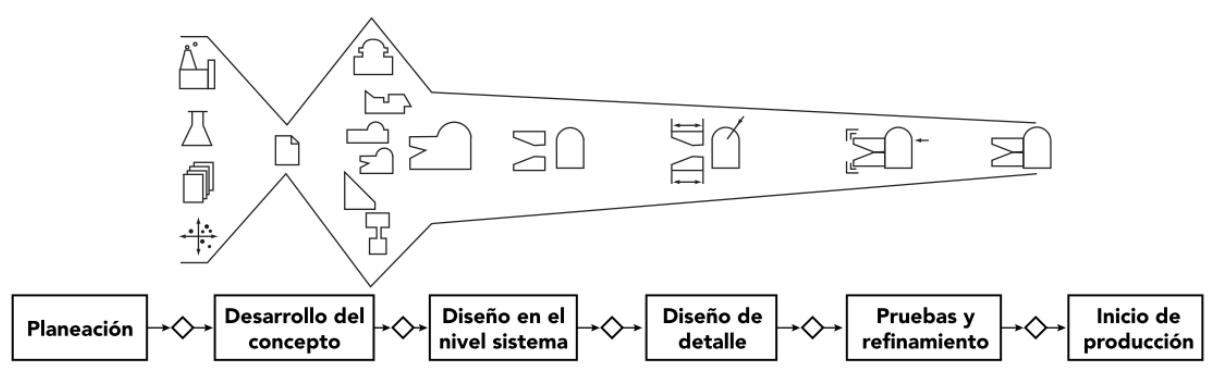

Fig. 2 Proceso genérico de desarrollo de product

Fuente: imagen obtenida del libro "Diseño y Desarrollo de Productos" (Ulrich \& Eppinger, 2003)

Un ejemplo de metodología desarrollada con la finalidad de facilitar herramientas para la mejora ambiental, es la propuesta por el IHOBE (2000), la cual consta de 7 etapas centradas en el ecodiseño. Esta ha sido aplicada principalmente en proyectos de mejora, donde se busca detectar los impactos ambientales y posteriormente desarrollar propuestas que busquen minimizarlos. Un ejemplo donde se han obtenido resultados exitosos es en la mesa de oficina GENIUS, desarrollada por Ofita, S.A.M.M., donde se logró una reducción del $52.32 \%$ del volumen de transporte, $4.5 \mathrm{~kg}$ por mesa en el consumo de acero y $5.6 \mathrm{~kg}$ por mesa en el uso de madera (IHOBE, 2000). 
Es importante resaltar que esta metodología no está centrada en la reutilización de los materiales y energías, siendo estos factores significativos para la propuesta que se busca realizar. Sin embargo, tiene relevancia ya que plantea un precedente en el uso del análisis del ciclo de vida del producto para la búsqueda de la reducción del impacto ambiental a lo largo de las distintas etapas del desarrollo de un producto.

Otro ejemplo de metodología es la propuesta en el artículo "Criterios de sostenibilidad en metodologías de diseño” (Alvarado Nieto, Roa López, \& Zuleta Ortiz, 2015) donde se expone la metodología ARZ, en la cual se detectaron 45 criterios enfocados a facilitar el diseño de productos sostentables. En la Fig. 3 se muestra los pasos correspondientes a la metodología ARZ.

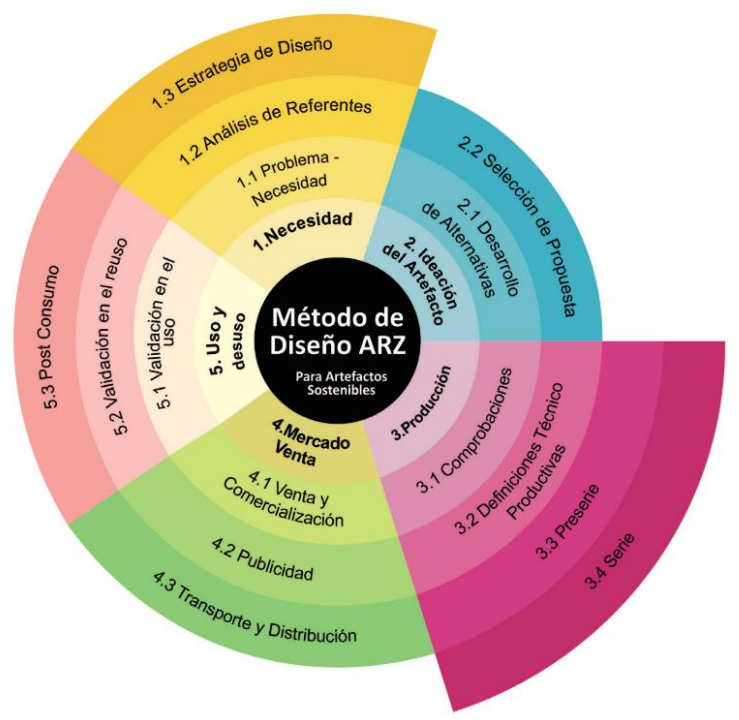

Fig. 3 Método de diseño ARZ para productos sostenibles

Fuente: tomado de (Alvarado Nieto et al., 2015)

Esta metodología se comparó con otras 7 por medio de una prueba piloto. Se les pidió a estudiantes de diseño industrial que crearan un objeto, donde cada uno de los grupos utilizó una metodología distinta. Las propuestas resultantes se mostraron a un grupo de posibles usuarios y clientes, siendo la desarrollada a base de la metodología ARZ la segunda favorita (Alvarado Nieto et al., 2015). Se resaltan factores relevantes de esta metodología, como la validación en el reúso y post consumo, siendo fundamentales dentro de la economía circular.

El diseño para la sustentabilidad (D4S), incluye factores ambientales, sociales y económicos (Crul \& Diehl, 2009). Esta metodología sostiene que para considerar un producto como sustentable, debe lograr innovar en las expectativas sociales, lograr una 
distribución equitativa de las ganancias a lo largo de toda la cadena de valor y disminuir el impacto a los ecosistemas que se encuentran relacionados a lo largo de todo el ciclo de vida del producto.

Algunos de los retos que se busca resolver a través del D4S, son: reducir la desigualdad en las ganancias, crear nuevas fuentes de empleo, uso de energías renovables, reducción y tratamiento de las emisiones industriales, valor agregado para compañías, proveedores y clientes (Stivale, 2020).

El diseño para la economía circular tiene como objetivo principal la reutilización de los materiales y energía para la generación de productos, buscando mantener un ciclo cerrado (Medkova \& Fifield, 2016). Para que esta metodología pueda ser implementada es necesario que, con cada producto, se desarrolle un nuevo modelo de negocio que asegure la circularidad de los materiales, convirtiéndose en una estrategia fundamental tanto para usuarios como para las compañías.

\section{Conclusiones}

Partiendo de estas experiencias previas, la problemática actual en materia medioambiental y manejo de residuos a nivel nacional y local, la necesidad de pasar a un modelo circular de producción y consumo y tomando como punto de partida las herramientas en las distintas metodologías; se plantea en la Fig. 4 la propuesta de metodología enfocada al desarrollo de alternativas de productos desechables sustentables.

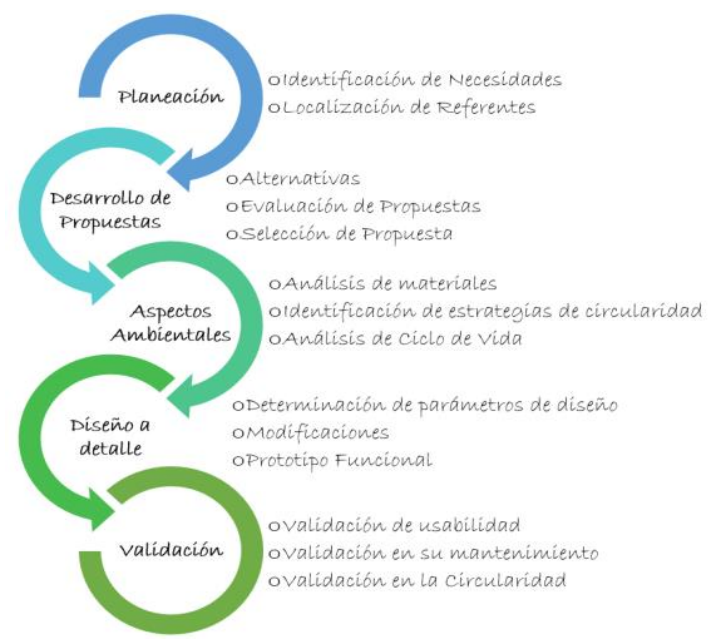

Fig. 4 Metodología para el análisis de ciclo de vida de materiales desechables Fuente: imagen de elaboración propia 
En este artículo se expone la necesidad de contar con una metodología que permita analizar las emisiones y residuos actuales de los productos desechables utilizados en el sector alimenticio, con la finalidad de poder desarrollar alternativas a los ya existentes.

La metodología planteada toma en cuenta las problemáticas generadas por las actuales formas de producción y consumo, la contaminación generada por las mismas y los objetivos planteados por la ONU, los cuales se convirtieron en referente para estrategias a nivel internacional, nacional y local.

Se analizaron diversas metodologías centradas en el medio ambiente, que nos permiten detectar aspectos clave en el proceso de diseño como lo son el ecodiseño, la economía circular y el análisis de ciclo de vida.

Esta propuesta metodológica forma parte de un proyecto en desarrollo, donde el ACV es fundamental para conocer los aspectos ambientales más relevantes, siendo estos los datos de entrada para la identificación de las necesidades y el desarrollo de las propuestas.

\section{Referencias}

Alvarado Nieto, G. A., Roa López, P. A., \& Zuleta Ortiz, D. L. (2015). Criterios de sostenibilidad en $\begin{array}{llll}\text { metodologías de } \quad \text { diseño. } & \text { Il12-132. }\end{array}$ https://doi.org/10.18566/iconofac.v11n17.a07

Bastidas Pacheco, G. A., \& Hernández, R. (2019). Cambio climático algunos aspectos a considerar para la supervivencia del ser vivo: revisión sistemática de la literatura. Revista Cuidarte, 10(3), 2135-2144. https://doi.org/10.15649/cuidarte.v10i3.664

Bernatene, M. del R., \& Canale, G. J. (2019). Innovación sustentable en Diseño a partir de la integración del análisis de Ciclo de Vida (ACV) con Cadenas Globales de Valor (CGV). Cuadernos Del Centro de Estudios de Diseño y Comunicación, 22(69), 151-174. https://doi.org/10.18682/cdc.vi69.1106

Crul, M., \& Diehl, J. C. (2009). Design for Sustainability A Step-by-step Approach. In United Nations Environment Programme. Retrieved from http://wedocs.unep.org/bitstream/handle/20.500.11822/8742/DesignforSustainability.pdf?sequenc $\mathrm{e}=3$ \&isAllowed $=y$

García, A., \& Cárcova, D. (2019). Del diseño industrial al design thinking . Perspectiva histórica de una disciplina en construcción. Centro de Estudios En Diseño y Comunicación, 94, 89-98.

Gobierno Municipal de Ciudad Juárez, Gobierno del Estado de Chihuahua, El Colegio de la Frontera Norte, Universidad Autónoma de Ciudad Juárez, Plan Estrategíco de Juárez, \& United Nations Development Programme. (2018). Juárez 2030 Plan para una Ciudad Sostenible. Retrieved from https://juarez2030.mx/wp-content/uploads/2018/11/juarez2030_291118.pdf

IHOBE. (2000). Manual práctico de ecodiseño. 182. Retrieved from 
http://www.valledelcauca.gov.co/agricultura/descargar.php?id=1756

INECC. (2018). Panorama de los Residuos en Mexico. In Instituto Nacional de Ecologia y Cambio Climatico. Ciudad de México.

Korhonen, J., Honkasalo, A., \& Seppälä, J. (2018). Circular Economy: The Concept and its Limitations. Ecological Economics, 143, 37-46. https://doi.org/10.1016/J.ECOLECON.2017.06.041

Medkova, K., \& Fifield, B. (2016). Circular Design - Design for Circular Economy. Lahti Cleantech Annual Review 2016, (February), 32-47.

SEMARNAT. (2016). Informe de la Situación del Medio Ambiente en Mexico. Compendio de Estadísticas Ambientales. Indicadores Clave y de Desempeño Ambiental. In Secretaría de Medio Ambiente y Recursos Naturales (2015th ed.). Retrieved from http://www.semarnat.gob.mx

Snyder, S. W. (2016). An introduction to commercializing biobased products: Opportunities, challenges, benefits, and risks. In RSC Green Chemistry (Vol. 2016-Janua). https://doi.org/10.1039/9781782622444-00001

Stivale, S. (2020). Los caminos del Diseño Sustentable y sus vinculaciones con la investigación en diseño. Cuadernos Del Centro de Estudios de Diseño y Comunicación, 21(80), 77-90. Retrieved from http://search.ebscohost.com/login.aspx?direct=true \&db=asu\&AN=129882213\&lang=es

Ulrich, K. T., \& Eppinger, S. D. (2003). Diseño y desarrollo de productos. In Diseño y desarrollo de productos. https://doi.org/10.1017/CBO9781107415324.004

United Nations Development Programme. (2015). Objetivos de Desarrollo Sostenible. Retrieved June 12, 2020, from https://www.undp.org/content/undp/es/home/sustainable-development-goals.html 